\title{
APLICACIÓN DE MÉTODOS DE CURADO Y SU INFLUENCIA EN LA RESISTENCIA A LA COMPRESIÓN DEL HORMIGÓN
}

\section{APPLICATION OF CURING METHODS AND THEIR INFLUENCE ON THE COMPRESSION STRENGTH OF CONCRETE}

\author{
Luis Daniel, Zambrano Navarrete ${ }^{1}$; Ronnie Jefferson, Alava Santos²; Wilter Enrique, Ruíz Párraga ${ }^{3}$; \\ Edgar Antonio, Menéndez Menéndez ${ }^{4}$
}

Recibido 10/10/2021: Aprobado: 17/12/2021

DOI: https://doi.org/10.51372/gacetatecnica231.4

\begin{abstract}
RESUMEN
El curado es de vital importancia en el hormigón ya que consiste en el fraguado y endurecimiento del mismo a través del tiempo, para obtener resistencias a la compresión óptimas. La presente investigación tuvo como objetivo determinar el método de curado que permita alcanzar la mayor resistencia a los 28 días. Se utilizaron tres tipos diferentes, estos son: hidratación continua, cubiertas húmedas y láminas de polietileno; para cada uno de ellos, se elaboraron nueve probetas cilíndricas aplicando la Norma Técnica Ecuatoriana del Instituto Ecuatoriano de Normalización NTE INEN 1576:2011, se realizaron los ensayos de resistencia a compresión, aplicando la NTE INEN 1573:2010, a los 7, 14 y 28 días con la finalidad de analizar el comportamiento de los testigos. La resistencia de diseño fue de $f^{\prime} c=21 \mathrm{MPa}$, los ensayos de resistencia a la compresión mostraron que el método con mayor beneficio para el hormigón fue el de cubiertas húmedas, alcanzando a los 28 días el valor de resistencia de 22,92 MPa con relación al f'c de diseño.
\end{abstract}

Palabras clave: curado del hormigón; resistencia a la compresión; método de hidratación continua del hormigón; métodos de cubiertas húmedas del hormigón

\footnotetext{
${ }^{1}$ Luis Daniel, Zambrano Navarrete. Estudiante de Ingeniería Civil. Universidad Técnica de Manabí. Ecuador. Correo: lzambrano4881@ utm.edu.ec ORCID: https://orcid.org/0000-0002-0829-8804

${ }^{2}$ Ronnie Jefferson, Alava Santos. Estudiante de Ingeniería Civil. Universidad Técnica de Manabí. Ecuador. Correo: Ralava9177@utm.edu.ec ORCID: https://orcid.org/0000-0003-2025-7914

${ }^{3}$ Wilter Enrique, Ruíz Párraga. Ingeniero Civil. MSc. Docente en la Universidad Técnica de Manabí. Ecuador. Correo: wilter.ruiz@utm.edu.ec ORCID: https://orcid.org/0000-0002-0045-9781

${ }^{4}$ Edgar Antonio, Menéndez Menéndez. Ingeniero Civil. MSc. Docente en la Universidad Técnica de Manabí. Ecuador.Correo: edgar.menendez@utm.edu.ec ORCID: https://orcid.org/0000-0001-7725-2706
} 


\begin{abstract}
Curing is of vital importance in concrete since it consists of its setting and hardening over time, in order to obtain optimum compressive strength. The objective of this research was to determine the curing method that allows reaching the highest resistance at 28 days. Three different types were used, these are: continuous hydration, wet covers and polyethylene sheets; for each of them, nine cylindrical specimens were made applying the NTE INEN 1576:2011, the compressive strength tests were carried out, applying the NTE INEN 1573:2010, at 7, 14 and 28 days in order to analyze the witness behavior. The design strength was $f^{\prime} c=21 \mathrm{MPa}$, the compressive strength tests showed that the method with the greatest benefit for the concrete was that of wet cover, reaching a strength value of $22.92 \mathrm{MPa}$ after 28 days with relationship to design $f^{\prime} c$.
\end{abstract}

Keywords: concrete curing; compressive strength; continuous hydration method of concrete; wet concrete cover methods

\title{
1. INTRODUCCIÓN
}

La importancia que tiene el hormigón en el medio constructivo es cada vez mayor, debido a que su maleabilidad permitiendo adaptarse a los diferentes tipos de construcciones en que se emplea. Además, presenta propiedades como la trabajabilidad y la durabilidad lo cual hacen de este material una herramienta eficaz a la hora de construir. Está compuesto por diferentes materiales, entre estos se encuentran principalmente el cemento, agregado fino (arena), agregado grueso (grava), agua y en ocasiones se emplean aditivos o adiciones; componentes que deben pasar por un correcto proceso de dosificación para poder brindar las características esenciales al hormigón, con el objetivo de que este alcance su máxima resistencia.

Todas estas características son esenciales para obtener un hormigón de calidad, sin embargo, usualmente se evalúa ésta en función de su resistencia a la compresión f'c al ser una característica mensurable; por ende, para que el hormigón alcance la máxima resistencia $f^{\prime} c$ la cual es especificada por el diseñador estructural, se debe someter a un adecuado proceso de curado inmediatamente después de ser fraguado. Uno de los factores clave en la ejecución de estructuras de hormigón es el curado, debido a que este proceso tiene por objeto brindar condiciones como la humedad y la temperatura que son esenciales para el desarrollo potencial de sus propiedades [1].

La Norma Ecuatoriana de la construcción, Estructuras de Hormigón Armado (NEC-SE-HM, 2015) [2] establece que "las superficies expuestas de hormigón que contienen cemento hidráulico deben mantenerse húmedas por el tiempo especificado en cada proyecto. Si no se dispone de esta especificación deben mantenerse húmedas por al menos de 5 a 7 días". Por ende, la importancia y eficacia que tiene el curado del hormigón es evidente, ya que este procedimiento ayuda a mantener húmedas las estructuras compuestas por este material 
dándole mejor calidad y por supuesto una vida útil más extensa.

En el Ecuador los métodos de curados están dados por la Norma Técnica Ecuatoriana del Instituto Ecuatoriano de Normalización NTE INEN 31242017 [3], en la que establece: para evitar la evaporación de agua del hormigón no endurecido, cubrir los especímenes inmediatamente después del acabado, preferiblemente con una placa o lámina no reactiva, no absorbente, de plástico resistente impermeable y durable. Cuando se usa una manta o tela húmeda como recubrimiento, no debe estar en contacto con la superficie del hormigón fresca y tener cuidado de mantener la tela húmeda hasta que los especímenes sean retirados de los moldes. Al colocar una lámina de plástico dicha la tela, la mantendrá húmeda.

Se ha observado que uno de los métodos más empleados en los procesos constructivos en el Ecuador es el de hidratación continua o "rociado de agua", sin embargo, mediante la presente investigación se desarrollaron especímenes de hormigón (testigos) a los cuales se le aplicaron métodos de curados de hidratación continua, cubiertas húmedas y láminas de polietileno, con la finalidad de obtener resultados que permitan estimar el método más favorable para alcanzar las resistencias deseadas en el diseño, para esto se realizó el ensayo de la resistencia a la compresión tal como lo establece la NTE INEN 1573:2010.

\section{DESARROLLO}

La Norma Ecuatoriana de la Construcción, Estructuras de Hormigón Armado (NEC-SE-HM, 2015) [2] define al hormigón como "Mezcla de cemento Portland o cualquier otro cemento hidráulico, agregado fino, agregado grueso y agua, con o sin aditivos". Por lo tanto, del control de calidad y proporción de la mezcla de estos materiales depende en gran medida las propiedades del hormigón. Así mismo, Nilson [4] describió al hormigón como "un material semejante a la piedra que se obtiene mediante una mezcla cuidadosamente proporcionada de cemento, arena y grava u otro agregado, y agua; después, esta mezcla se endurece en formaletas con la forma y dimensiones deseadas".

Éstas aseveraciones son corroboradas por Abanto [5] quien señaló que "el cemento y el agua reaccionan químicamente uniendo las partículas de los agregados, constituyendo un material heterogéneo. Algunas veces se añaden ciertas sustancias, llamadas aditivos, que mejoran o modifican las propiedades del hormigón". La materia prima constituyente del hormigón, influyen en la resistencia a la compresión, además es importante tener presente la relación agua/cemento (A/C) debido a que es la encargada de proporcionar al material la resistencia necesaria [6]. 
$\overline{\text { Para la fabricación de hormigones estructurales se utilizan únicamente cementos hidráulicos, }}$ los cuales reaccionan químicamente cuando se les añade cierta cantidad de agua. El cemento es un polvo muy fino que posee un color grisáceo, se obtiene mediante procesos especiales de la combinación de arcillas, yesos y calizas [7]. Mientras que el agua es el encargado de hidratar las partículas del cemento, para formar la pasta aglutinante. Según la NEC-SE-HM [2] si el agua es apta para el consumo humano es buena para hacer hormigón, pero cuando se tienen dudas sobre su calidad se deben aplicar los siguientes dos criterios para su aceptación:

- La resistencia a la compresión de cubos de mortero ensayados de acuerdo con la norma NTE INEN 488 ó ASTM C109 (American Society for Testing and Materials, por sus siglas en ingles) y fabricados con el "agua dudosa" sea por lo menos el $90 \%$ de la resistencia a la compresión de cubos de mortero fabricados con agua aceptable para hacer hormigón, comparados a la edad de 7 días.

- La desviación del testigo en el ensayo de tiempo de fraguado ejecutado en concordancia con la norma ASTM C191, se encuentre dentro del rango de 1 hora antes hasta 1 hora 30 minutos después.

Adicionalmente se tienen los agregados, "como agregados o áridos para hormigones pueden tomarse en consideración todos aquellos materiales que, poseyendo una resistencia propia suficiente (resistencia del grano), no perturban ni afectan el proceso de endurecimiento del cemento hidráulico" [8]. Estos agregados naturales se clasifican en gruesos y finos, es decir, los agregados gruesos o gravas no pasan por el tamiz \#4 y son los que poseen mejor propiedad adherente con la pasta del cemento, mientras que los agregados finos o arenas si pasan por el tamiz \#4.

Mientras que el aditivo se refiere a las sustancias que son añadidas a los componentes fundamentales del hormigón con el objetivo de mejorar o modificar sus propiedades. Estas sustancias se añaden cuando son requeridas en el proyecto, ya que mejoran las condiciones de trabajo y ayudan a reducir los costos de producción. Se debe tener precauciones en el uso de aditivos, debido a que "puede ocurrir que mientras una propiedad mejore favorablemente, otra cambie en forma adversa. Por ejemplo, la durabilidad del hormigón se incrementa con la incorporación del aire, pero su resistencia disminuye" [5]. El hormigón como material presenta varias propiedades, tales como la trabajabilidad, durabilidad, cohesividad y resistencia, son importantes para la calidad del mismo, por tanto, se debe de tener criterios técnicos en la selección de los materiales que lo conforman, posterior a ello se realiza una adecuada dosificación aplicando una serie de códigos y normas, como el American Concrete Revista Gaceta Técnica. Artículo de Investigación. 23(1), 35-47, enero-junio, 2022 
Institute del ACI-211.1.

\subsection{Resistencia a la Compresión del Hormigón}

La resistencia a la compresión es la característica mecánica principal que posee el hormigón, esta hace referencia a la capacidad de soporte de carga por unidad de área que este posee, la cual se expresa en términos de esfuerzo. Dicha resistencia $f^{\prime} c$, utilizada en diseño estructural, se obtiene mediante términos probabilísticos, es decir, que simplemente un pequeño porcentaje de las muestras que normalmente se encuentran en el 5\% alcanzan resistencias inferiores a la especificada en el diseño, por tanto la resistencia media de los especímenes $(\mathrm{fm})$ siempre resultará mayor que la resistencia característica [9]. La resistencia del hormigón no puede probarse en condición plástica, debido a que el procedimiento frecuente consiste en tomar especímenes durante el mezclado los cuales después de curados se someten a pruebas de compresión [5].

La Norma Técnica Ecuatoriana NTE INEN 1576:2011 [10] indica que cuando el hormigón está fresco se le realizan los ajustes necesarios, para proceder a muestrearlo y determinar las características físicas que posee, todo esto de acuerdo a un procedimiento normalizado. Posteriormente se toman muestras de hormigón en moldes especificados según el proyecto, estos moldes pueden ser cilindros o vigas, de acuerdo a los procedimientos indicados por esta norma. Así mismo, la NTE INEN 1573:2010 [11] menciona que se debe tener cuidado con la interpretación del significado que implica la determinación de la resistencia a compresión con los procedimientos utilizados por este método de ensayo, debido a que la resistencia no es una propiedad fundamental o intrínseca del hormigón elaborado con materiales dados.

Además, los valores obtenidos dependerán de la geometría del espécimen (tamaño y forma), dosificación, procedimientos de mezclados, métodos de muestreos y demás factores que influirán durante el proceso de curado respectivo del hormigón. También es necesario mencionar que existen diversos factores que afectan la resistencia a la compresión del hormigón, tales como: la relación agua-cemento (A/C), el contenido de cemento, el tipo de cemento, las condiciones de curado, tipo de agregado, mal uso de los métodos de compactado.

Es necesario realizar los adecuados procedimientos que forman parte del proceso del diseño del hormigón, tomando en cuenta todas las normas que intervienen en este proceso, para lograr conseguir resultados favorables y adecuados para que el hormigón sea de buena calidad y mejore sus propiedades. 


\subsection{Métodos de Curado}

De acuerdo con el ACI 308-92 [12] "el curado consiste en mantener un contenido satisfactorio de humedad y temperatura en el hormigón recién colado para que puedan, así, desarrollarse las propiedades deseadas". Es decir, que se debe curar al hormigón de una manera adecuada para que las propiedades como la resistencia y la durabilidad se desarrollen absolutamente. Existen diversos procedimientos, métodos y materiales para realizar el curado del hormigón, los cuales cumplen el mismo objetivo, el de garantizar el mantenimiento de humedad y temperatura para obtener las propiedades deseadas en el hormigón. Es decir que mientras mayor cantidad de humedad se retenga, mayor es la eficiencia del método de curado.

La NEC-SE-HM [2] y el ACI 308-92 [12] mencionan que existen dos sistemas para conservar la humedad y buena temperatura del hormigón, el primero de ellos seria mediante el curado con agua, que consiste en la aplicación continua de agua, ya sea por anegamiento, aspersión, cubiertas de materiales saturados, como lo son las mantas de yute o algodón, alfombras, aserrín, entre otros. Mientras que el otro sistema seria con materiales selladores el cual consiste en evitar la pérdida excesiva de agua en la superficie del hormigón, con la ayuda de materiales como lo son las hojas plásticas, los papeles impermeables o mediante la aplicación de compuestos de curado formadores de membranas.

El curado mediante hidratación continua o aspersión [12] se realiza mediante la aplicación del uso de mangueras, sin embargo, se debe tener cuidado de no provocar erosión en la superficie que se está aplicando agua. Este procedimiento no es recomendable cuando la superficie del hormigón se encuentra seca, debido a que, este método se lo aplica siempre que la superficie del hormigón este más fría que la atmosfera dentro del recinto con el objetivo de que se genere una película de humedad sobre la superficie. Éste método es muy fácil de ejecutar y se pueden conseguir resultados muy buenos, Sin embargo, presenta inconvenientes de tipo practico entre los cuales resaltan en que la intermitencia o aplicación ocasional puede conducir a obtener un curado deficiente [13].

Mientras que, la influencia del curado utilizando cubiertas de arpillera como el yute tiene gran incidencia en las propiedades del hormigón como lo son la resistencia y durabilidad. El proceso de curado debe empezar en cuanto el hormigón se endurece lo suficiente para prevenir la erosión de la superficie y mantener húmedo el material para obtener resultados más efectivos [14]. Manobanda [1] enfatizó que este tipo de curado cuenta con el riesgo de absorber la humedad interna del hormigón al no ser humedecidos frecuentemente, produciéndose así una deficiencia en el curado. Por tal razón estos materiales absorbentes 
deben ser humedecidos periódicamente para evitar deficiencias en el hormigón.

Mientras que para el curado mediante láminas de polietileno la película plástica debe tener un peso ligero con un espesor de $0,10 \mathrm{~mm}$, se debe tener cuidado con no rasgar o dañar este material, ya que interrumpiría la continuidad del curado. La película negra tiene la ventaja en clima frio por su absorción de calor, mientras que la transparente tiene poco efecto en la absorción del calor [12]. La película blanca es más costosa, pero los rayos solares se reflejan considerablemente en ella. Se recomienda este método de curado para climas cálidos, ya que ayudan a proteger las estructuras de factores externos que puedan alterar las propiedades del hormigón, además concluyen que es una de las mejores opciones al momento de realizar el curado en obra [15].

\section{METODOLOGÍA}

Se inició con la selección de los materiales que conforman el hormigón, según el Plan de Desarrollo y Ordenamiento Territorial de Manabí 2015 - 2024 [16], existen alrededor de 50 canteras en Manabí, de estas se escogió a Megarok S.A., donde se encuentra la mayor producción mensual de materiales pétreos de la provincia, la beta de esta empresa es de tipo basáltica [17]. Se empleó el cemento hidráulico portland tipo GU en presentación de 50kg. Luego se realizó la dosificación correspondiente de los materiales para obtener el diseño de la mezcla de hormigón, tomando como referencia el valor mínimo de $f^{\prime} c=21 \mathrm{MPa}$ de acuerdo con la NEC-SE-HM [2], aplicando la normativa del Instituto Americano de Concreto (ACI 211.1) y la NTE INEN 1855-2 utilizando una relación A/C de 0,49 según lo indicado en la Tabla 2 de la misma norma [18], con grado de exposición moderado. A continuación, se presenta los valores respectivos de la dosificación de la mezcla de hormigón para un metro cubico evidenciado en la Tabla 1.

Tabla 1. Dosificación de la mezcla de hormigón. Fuente: los autores

\begin{tabular}{cccc}
\hline Descripción & Pesos $(\mathbf{N})$ & Densidades $\left(\mathbf{k g} / \mathbf{m}^{\mathbf{3}}\right)$ & Volumen $\left(\mathbf{m}^{\mathbf{3}}\right)$ \\
\hline Cemento & 3407,45 & 1198 & 0,290 \\
Agua & 1484,73 & 1003 & 0,151 \\
Agregado Fino & 9295,92 & 1441 & 0,658 \\
Agregado Grueso & 9827,01 & 1400 & 0,716 \\
Peso Teórico (kg) & 24015,01 & & \\
A/C & & 0,49 & 1,82
\end{tabular}

Una vez que se obtuvo la mezcla de hormigón se realizó la medición de la trabajabilidad mediante el cono de Abrams [18], obteniendo un asentamiento de $60 \mathrm{~mm}$ como se observa en la Figura 1, de acuerdo a la NTE INEN 1578:2010 [18], para tal paso se humedecieron los 

DEL HORMIGÓN

equipos para evitar restarle agua a la mezcla. Luego se procedió a limpiar y cubrir ligeramente con aceite mineral a las probetas en su interior para la preparación de los cilindros de hormigón como lo establece NTE INEN 1576:2011 [10].

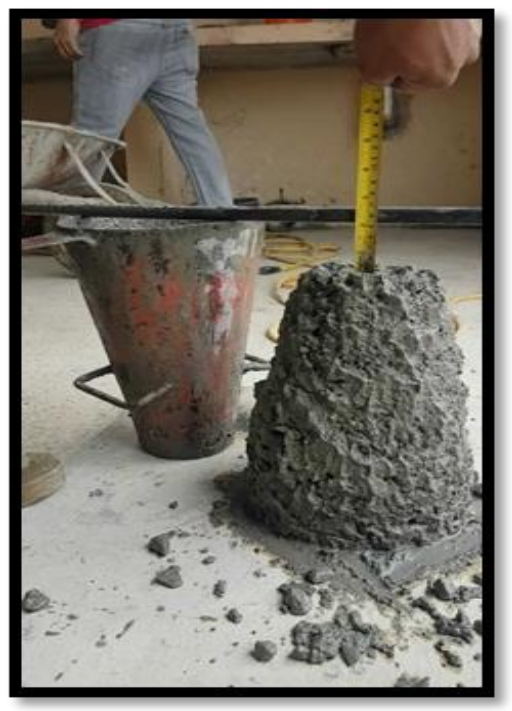

Figura 1. Medición de asentamiento mediante el cono de Abrams. Fuente: los autores

\subsection{Curados Empleados}

\section{Hidratación continua}

42 Los nueves espécimen bajo la aplicación de este método de curado se rociaron con agua a través del uso de mangueras 3 veces al día, colocadas en un ambiente abierto como se observa en la Figura 2. Se procedió a regar con agua potable de acuerdo con los siguientes horarios establecidos: primer riego por la mañana 9:00, segundo riego por la tarde, 14:00 y el tercer riego por la noche, 19:00.

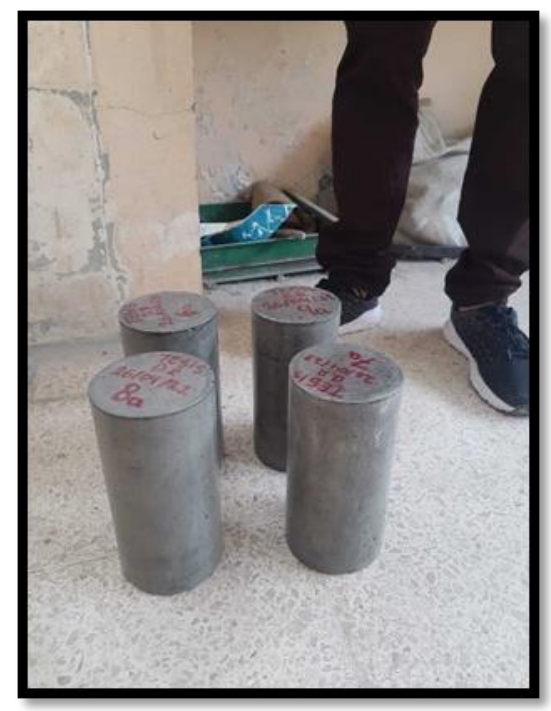

Figura 2. Especímenes curados mediante hidratación continua. Fuente: los autores 
Cubiertas húmedas

Nueve de las probetas realizadas fueron envueltos completamente en tejido de yute de 50 x 75 $\mathrm{cm}$, para que esta retenga la humedad mediante un riego con agua usando una manguera, las cuales fueron colocadas en un ambiente abierto como se observa en la Figura 3. Se procedió a hidratar con agua potable de la misma manera que se utilizó con la hidratación continua de acuerdo con los siguientes horarios establecidos: primer riego por la mañana, 9:00, segundo riego por la tarde, 14:00 y el tercer riego por la noche, 19:00.

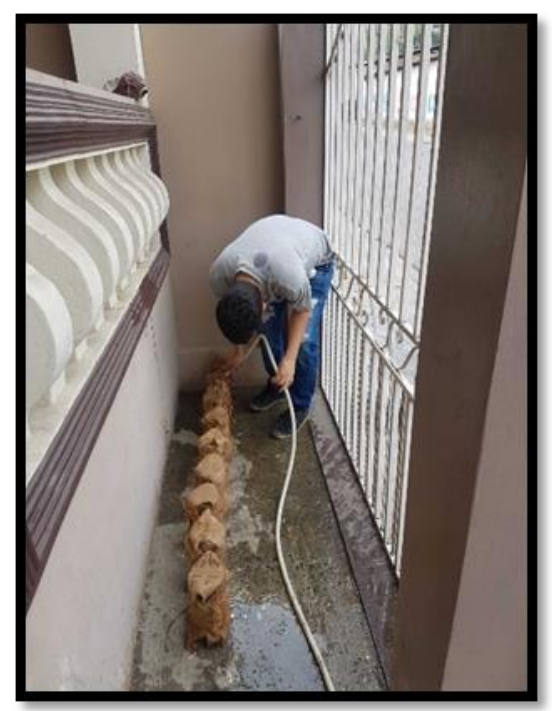

Figura 3.Especímenes curados mediante cubiertas húmedas (Yute). Fuente: los autores

\section{Láminas de polietileno}

Las ultimas nueve probetas fueron revestida con una lámina de polietileno negra de 50 x 75 cm para que esta mantenga la humedad y a su vez absorba el calor ya que esta no admitirá más hidratación externa tan solo la ya existente en el hormigón, estos especímenes fueron colocados en un ambiente cerrado durante los días de ensayo (ver Figura 4).

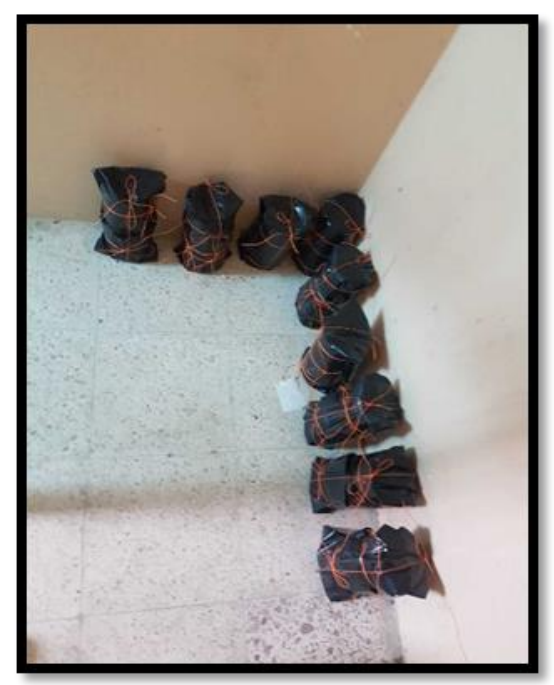

Figura 4. Especímenes curados mediante lámina de polietileno. Fuente: los autores 
Se realizó los ensayos de la resistencia a la compresión a los primeros 7 días a 3 probetas por cada tipo de curado objeto de estudio. En fechas posteriores, es decir a los días 14 y 28, se realizaron los ensayos correspondientes para obtener la resistencia de los testigos ensayados, como se observa en la Figura 5.

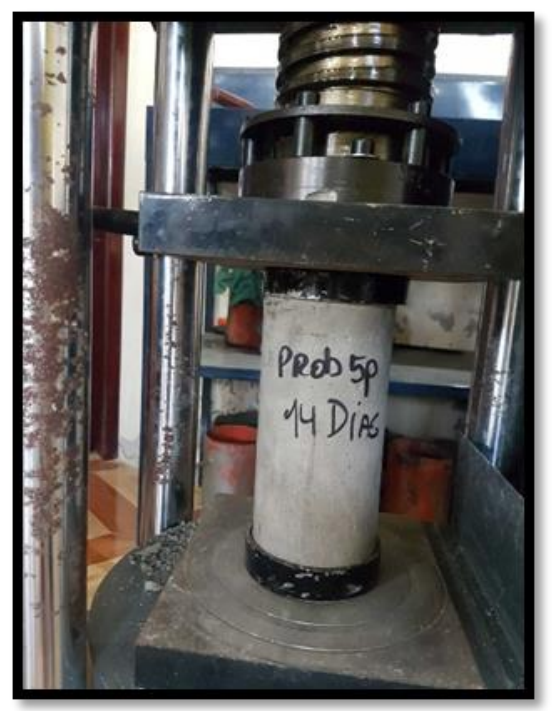

Figura 5. Ensayo de resistencia a compresión. Fuente: los autores

\section{RESULTADOS}

Los resultados obtenidos de los ensayos de resistencia a la compresión para las edades de rotura de 7 días, 14 días y 28 días, para los diferentes tipos de curado correspondientes a cubiertas húmedas, hidratación continua y láminas de polietileno, se muestran en la Tabla 2.

Tabla 2. Resistencias a la compresión de los diferentes métodos de curado, en MPa. Fuente: los autores

\begin{tabular}{llll}
\hline Tipo de curado & $\mathbf{7}$ días & 14 días & 28 días \\
\hline Cubiertas húmedas & 20,46 & 21,71 & 22,92 \\
Hidratación continua & 19,15 & 20,84 & 22,31 \\
Láminas de polietileno & 18,33 & 19,86 & 21,81 \\
\hline
\end{tabular}

Se observa que, para la edad de 7 días, en relación a los métodos de curados, la cubierta húmeda alcanzó un valor de 20,46 MPa, el método de láminas de polietileno obtuvo el valor más bajo, es decir 18,33 MPa. Para las probetas ensayadas a los 14 días el mayor valor que se obtuvo fue mediante las cubiertas húmedas con 21,71 $\mathrm{MPa}$, seguida por la hidratación continua con el valor de 20,84 MPa y el valor más bajo corresponde al curado con láminas de polietileno con un valor de 19,86 $\mathrm{MPa}$.

En los ensayos de resistencia a la compresión a los 28 días se obtuvieron los valores promedios de las probetas ensayadas, como criterio de aceptación para estos resultados se tenía en cuenta el rango entre $f^{\prime} c=21 \mathrm{MPa}$ y $f^{\prime} \mathrm{cm}=23 \mathrm{Mpa}$ obteniéndose los valores que se 
muestran en este párrafo según el tipo de curado. El método a través de cubiertas húmedas, alcanzó la resistencia a la compresión de 22,92 MPa, que representa el 109,1\% con relación a la resistencia de diseño, considerándose el valor más alto con relación a los tres tipos de curados que se emplearon en la investigación. El curado de hidratación continua obtuvo un valor de 22,31 $\mathrm{MPa}$, que representa el 106,2\% y finalmente para el método de lámina de polietileno se obtuvo un valor de 21,81 MPa, que representa el 103,9\%. En la Figura 6 se muestra la curva de resistencia a la compresión con los valores obtenidos a los 28 días con los tres tipos de curados empleados.

\section{CURVA DE RESISTENCIA A LA COMPRESIÓN}

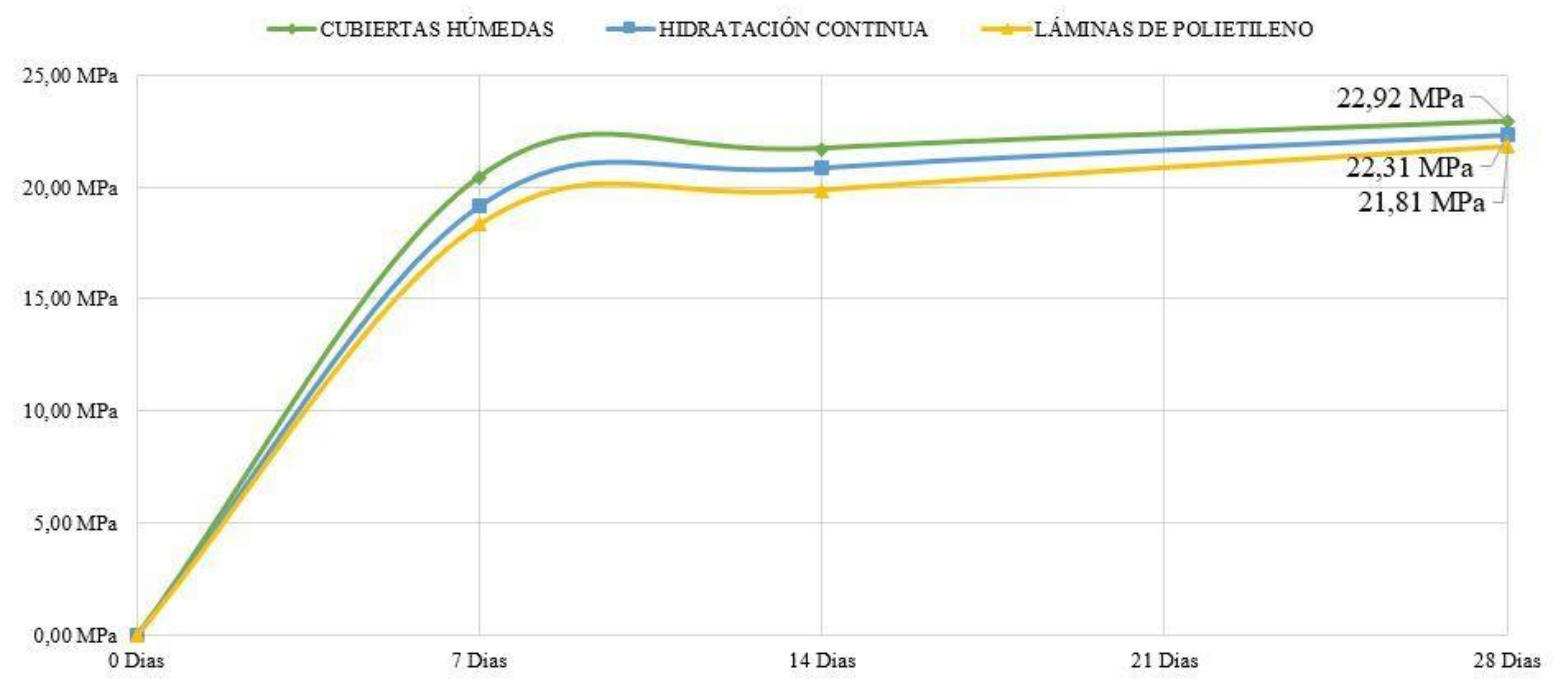

Figura 6. Curva de la resistencia a la compresión. Fuente: los autores

\section{CONCLUSIONES}

Los tres tipos de curados empleados en esta investigación demuestran que son factibles utilizarlos, ya que los valores de resistencia a la compresión a los 28 días, indican ser mayores a la resistencia de diseño. Por la experiencia y la información bibliográfica analizada, el curado más recomendado que se puede aplicar en obra es el método de cubiertas húmedas ya que mantiene al hormigón hidratado por mayor tiempo y esto permite alcanzar las resistencias esperadas. De acuerdo con el método experimental utilizado en esta investigación, se logró determinar que el curado del hormigón si influye significativamente en la resistencia a la compresión, lo cual se observó desde las primeras roturas (día 7) ya que el hormigón aumento de una forma acelerada su resistencia hasta terminar con los ensayos (día 28).

\section{REFERENCIAS}

[1] C. Manobanda, "El curado del hormigón y su incidencia en las propiedades mecánicas finales", Tesis BA, Carrera de Ingeniería Civil, Universidad Técnica de Ambato, 
Ambato, 2013.

[2] MINISTERIO DE DESARROLLO URBANO Y VIVIENDA, "Norma Ecuatoriana de la Construcción (NEC-2015)”, NEC SE HM., Quito, 2015.

[3] NTE INEN 3124:2017-3. Instituto Ecuatoriano de Normalización. "Norma Técnica Ecuatoriana. Hormigón. Elaboración y curado de especímenes de ensayo en el laboratorio", Quito, 2017.

[4] A. Nilson, "Diseño de estructuras de concreto", Duodécima ed., Bogota: Mc Graw Hill, 2001.

[5] F. Abanto Castillo, “Tecnología del concreto", Lima-Perú: Editorial San Marcos, 2009.

[6] L. León y M. Hernández, "Comparación de los valores de resistencia a compresión del hormigón a la edad de 7 y 28 días", Revista Arquitectura e Ingenieria, vol. 10, nº 1, pp. $1-9,2016$.

[7] M. Sanjuán y S. Chinchón, "Introducción a la fabricación y normalización del cemento Portland”, San Vicente del Raspeig: Universidad d'Alacant: UNE, 2004.

[8] D. Sánchez de Guzmán, “Tecnologia del concreto y del mortero”, Quinta edición, Santafé de Bogota: Bhandar Editores Ltda, 2001.

[9] M. Romo Proaño, “Temas de hormigón armado”, Quito, ESPE, 2008.

[10] NTE INEN 1576:2011, Instituto Ecuatoriano de Normalización, "Norma Técnica Ecuatoriana. Hormigón de cemento hidráulico. Elaboración y curado en obra de especímenes para ensayo", Quito, 2011.

[11] NTE INEN 1573:2010, Instituto Ecuatoriano de Normalización, "Norma Técnica Ecuatoriana. Hormigón de cemento hidráulico. Determinación de la resistencia a la compresión de especímenes cilíndricos de hormigón de cemento hidráulico", Quito, 2010 .

[12] AMERICAN CONCRETE INSTITUTE (ACI), "Práctica estándar para el curado del concreto", ACI 308-92, Instituto Mexicano del Cemento y del Concreto, A.C., México, 1994.

[13] V. Villegas, "Ventajas comparativas del curado de concreto con agua vs curado de concreto con productos químicos en la ciudad de Huaraz", Tesis BA, Escuela de Ingeniería Civil, Universidad Nacional Santiago Antúnez de Mayolo, Huaraz, 2012.

[14] S.L., Tejijut, "Utilización del tejido de yute para el curado del hormigón” Disponible en: https://www.deyute.com/sec/es/news/utilizacion-del-tejido-de-yute-para-el-curado-delhormigon/40, 2013

[15] Z. Jiménez y D. Ordoñez, “Análisis de la influencia de las técnicas empleadas para el curado de cilindros de concreto hidráulico sobre la resistencia a la compresión", Tesis BA, Departamento de Civil y Ambiental Barranquilla, Universidad de la Costa, 
Barranquilla, 2021.

[16] Consejo Provincial de Manabí, "Plan de Desarrollo y Ordenamiento Territorial de Manabí 2015 - 2024 Provincia del Milenio", 2015.

[17] Megarok S.A, “Megarok S.A”, Dsponible en: http://megarok.com.ec/web/, 2021

[18] NTE INEN 1855-2, Instituto Ecuatoriano de Normalización, "Norma Técnica Ecuatoriana. Hormigones. Hormigón Preparado en Obra. Requisitos”, Quito, 2015. 